\title{
Effects of Hydrogen on InP Light-Emitting Devices Etched in a Methane-Hydrogen Environment
}

\author{
Patricia Beck ${ }^{1}$, Dennis Derickson ${ }^{2}$, Forrest Kellert ${ }^{2}$ and Tim Bagwell ${ }^{2}$ \\ ${ }^{1}$ Hewlett-Packard Laboratories, Palo Alto, CA 94306, USA \\ ${ }^{2}$ Hewlett-Packard Lightwave Operations, Santa Rosa, CA 95403, USA
}

\section{Introduction}

Research has been conducted on the physical damage and hydrogenation effects during RF plasma exposure $(1,2)$ and epitaxial growth $(3,4)$ in the III-V material system. Device consequences of this damage or chemical alteration have received less attention, particularly in active light emitting devices. This paper discusses these effects for lasers and edge emitting light-emitting diodes (EELEDs) which use a ridge waveguide structure. By using analysis techniques such as SIMS we have concluded that methane-hydrogen reactive ion etching (RIE) of InP induces hydrogen levels in an active device which are high enough to significantly alter the device properties. The decrease in light output is substantial, but subsequent annealing times as short as $1 \mathrm{~min}$. at $430^{\circ} \mathrm{C}$ can restore power dramatically.

\section{Ridge Waveguide Fabrication}

Fig. 1 shows the general device structure analyzed in this work. The substrate is $n$-type InP with a lattice matched $\mathrm{In}_{1-\mathrm{X}} \mathrm{Ga}_{\mathrm{X}} \mathrm{As} \mathrm{s}_{\mathrm{Y}} \mathrm{P}_{1-\mathrm{Y}} \mathrm{QW}$ active region for the lasers and bulk InGaAsP active region for the EELEDs. The contact layer is heavily doped and bandgap-graded to InGaAs.

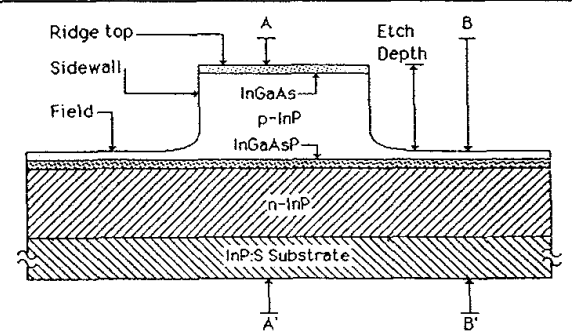

Fig. 1. Material layers. Section $A-A^{\prime}$ is through the ridge. Section B-B' is through the field.

An EELED has gain and absorber regions while a laser has only a gain region. Each waveguide is fabricated by the same basic process. When current flows between the ridge top contact and the back side of the substrate, light is emitted along the current flowing region. In addition to gain guiding, the light is guided by index differences between $\operatorname{InP} / \operatorname{In}_{1-X} \mathrm{Ga}_{\mathrm{X}} A s_{Y} \mathrm{P}_{1-\mathcal{Y}}$ epitaxial layers in the vertical direction and the index step from the ridge waveguide to the barrier dielectric along the length of the ridge (5).

The ridge confinement structure is produced by first patterning the substrate with an etch resistant material $(6$, 7) and then RIE etching in an environment consisting of $15 \%$ methane in hydrogen at $400 \mathrm{~V}$ and $100 \mathrm{~W}$ for two to three hours. An increased etch rate may be obtained with the addition of argon to the mix. In the RF excited plasma the methane and hydrogen are thought to split apart to form methyl and hydrogen groups.

$$
\begin{array}{lll}
\mathrm{CH}_{4} & \left.\left.-{ }^{E}-\right\rangle\right) & \mathrm{xCH}_{3}{ }^{*}+\mathrm{yCH}_{2}{ }^{*}+\mathrm{zCH}^{*}+\text { ions } \\
\mathrm{H}_{2} & \left.\left.{ }^{\mathrm{E}}{ }_{-}\right\rangle\right) & \mathrm{x}^{\prime} \mathrm{H}_{2}^{+}+\mathrm{y}^{\prime} \mathrm{H}^{*}+\text { protons }
\end{array}
$$

When reacted with the InP-based compounds, the end products are methyl-indium or methyl-gallium compounds and hydrides such as phosphine or arsine, reversing the OMVPE process (8).

$$
\begin{aligned}
& \left.\mathrm{In}+3 \mathrm{CH}_{3}^{*}-\rightarrow\right) \operatorname{In}\left(\mathrm{CH}_{3}\right)_{3} \\
& \mathrm{P}+3 \mathrm{H}^{*} \rightarrow \gg \mathrm{PH}_{3}
\end{aligned}
$$

The side wall etch rate is lower than that of the field. Therefore, side walls are exposed to a hydrogen rich environment (and physical damage) for most of the etch time while the field is removed to a predetermined depth above the active region by active monitoring.

Before removal from the RIE chamber, the sample is exposed to an oxygen plasma to remove the polymer film which has formed during the methane-hydrogen etch. The ridge is coated with a dielectric material. Then the ridge top is etched free of the dielectric and original masking material. Metal is deposited to contact the top of the ridge ( $p$-side). After thinning the substrate, metal is applied to the back $n$-side. The wafer is annealed and cleaved into devices. The final device structure is shown in Fig. 2.

\section{Experimental Results}

\section{Decreased Light Output}

As originally fabricated, both lasers and EELEDs exhibited poor output power. EELEDs with 4- $\mu \mathrm{m}$-wide ridges and $800-\mu \mathrm{m}$-long gain regions produced approximately $100 \mu \mathrm{W}$ at a drive current of $200 \mathrm{~mA}$. Laser structures of various lengths from the same wafer also showed high threshold current and poor quantum efficiency. Elevated temperature strife testing revealed a slow increase in power over time, for constant drive current. The fabrication anneal was performed at $400^{\circ} \mathrm{C}$ for $30 \mathrm{sec}$. This was found to be insufficient to both anneal the ohmic metals and to relieve damage. 


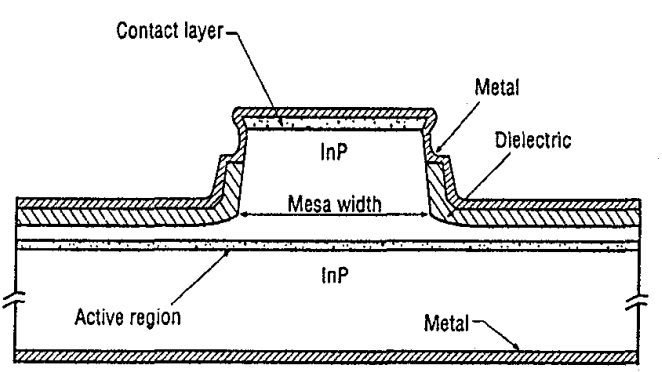

Fig. 2. Cross section of etched ridge structure.

\section{Experimental Heat Treatments}

Subsequent higher temperature treatments on a 4- $\mu \mathrm{m}$-wide EELED increased device output power four to five times with little change in contact resistance. Below $300{ }^{\circ} \mathrm{C}$ noticeable power increases are measured in hours, while above $400{ }^{\circ} \mathrm{C}$ the same changes are achieved in minutes. The optimum required heat treatment time varies logarithmically with temperature (Fig. 3), evidence of diffusion processes at work.

Fig. 4 shows the optical output power for a non-heat-treated device compared with the same device annealed at $400^{\circ} \mathrm{C}$, as a function of time. The figure also shows that it is possible to degrade the device with an extended anneal. Wider ridges with similar gain lengths exhibited greater initial output power than narrower ridges and showed less percentage improvement from the temperature treatments. Output from an $8-\mu \mathrm{m}$-wide ridge increased two to three times while that from a $100-\mu \mathrm{m}$-wide ridge showed no quantifiable difference. Although both bulk and QW material were affected, the QW material showed smaller improvements after the heat treatments.

\section{Material Analysis}

To study the effects of the methane-hydrogen etching, SIMS analysis for zinc and hydrogen was performed on bulk and QW samples. Referring to Fig. 1, Sample I is

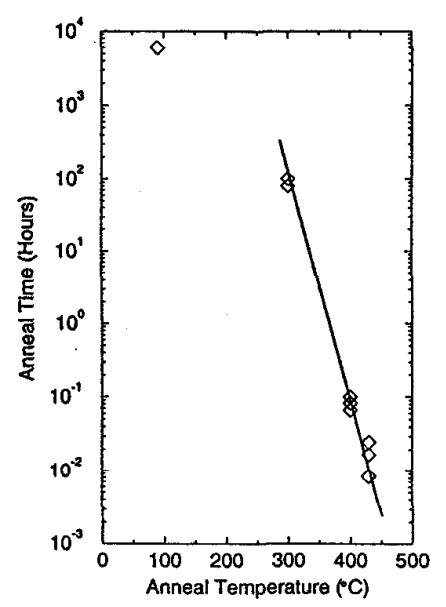

Fig. 3. Anneal time for power recovery vs. temperature.

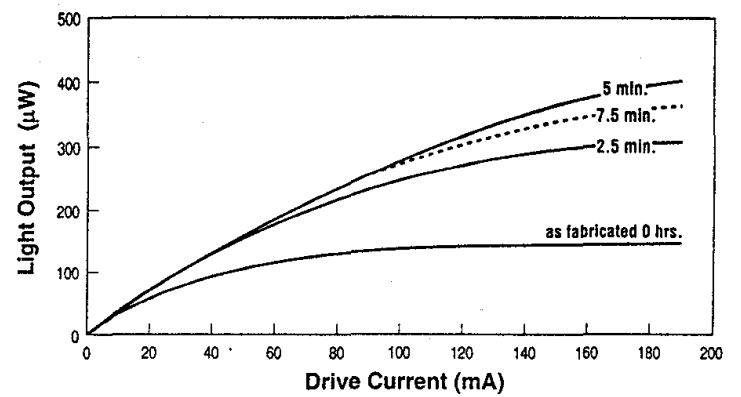

Fig. 4. Output power vs. anneal time at $400{ }^{\circ} \mathrm{C}$ for an unheatsunk EELED with a $4-\mu \mathrm{m}$-wide ridge.

taken through Section $A-A^{\prime}$, samples II-V are through Section B-B'. The following are included:

(I) Before etching - full structure (bulk material)

(II) Etched without Argon in the mix (bulk)

(III) Etched without Ar in the mix (QW)

(IV)Etched with Ar in the mix (QW),

(V) Etched without Ar, annealed at $430^{\circ} \mathrm{C}, 2 \mathrm{~min}$. (bulk).

Profiles show elevated hydrogen levels extending up to $4 \mu \mathrm{m}$ into the substrate from the exposed surface (Fig. 5) when compared with the as-grown structure (Fig. 6). After a short high temperature anneal (Fig. 5) the hydrogen returned to as-grown levels except in the active area, which may have a slight affinity for hydrogen, and the interface regions. Particularly obvious is a spike at the undoped epitaxial InP/substrate interface. Since the interface is $n$-InP/n-InP, a smooth diffusion profile was expected. The hydrogen appears to be passivating interface defects which anneal out at much higher temperatures. The "after-etch" plots are taken from the field of the sample (refer to Fig. 1) because $4-\mu \mathrm{m}$-wide ridges are too narrow for effective SIMS.

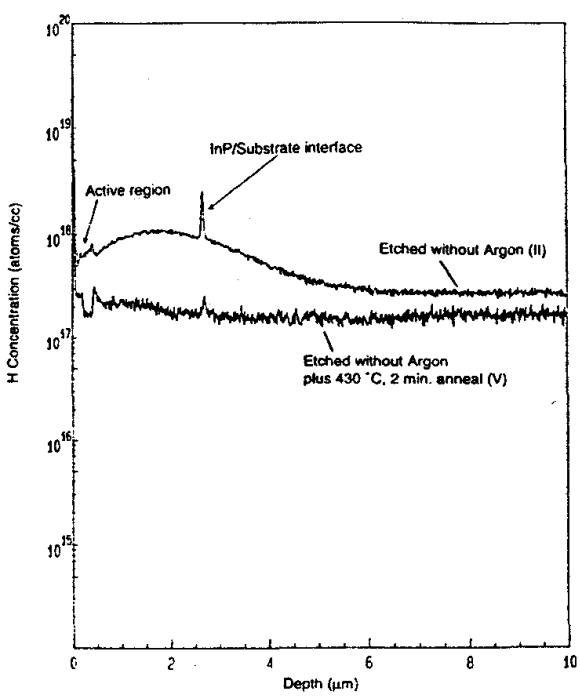

Fig. 5. Hydrogen SIMS data from samples "as etched" (II) and "etched plus annealed" (V). 


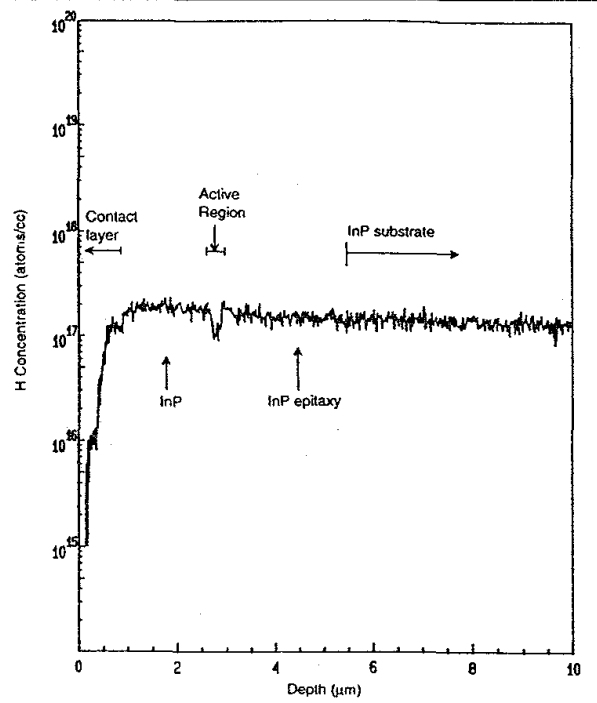

Fig. 6. Hydrogen SIMS data in the "as grown" sample (I).

The amount of hydrogen in InGaAsP relative to InP may not be valid as read directly from the figures because InP was the SIMS reference. The overall changes are valid and values should be compared between regions of similar composition.

These samples were etched in a method identical to the tested light-emitting devices, including the additional oxygen plasma clean before further processing. We believe that the heating which occurs during this step, in the non-hydrogen environment, decreases the surface hydrogen concentration. This "anneal" would explain why the peak concentration is not at the surface in Fig. 5.

The addition of argon to the etch increased the etch rate through physical bombardment and decreased the time that the sample was exposed to the hydrogen plasma. Argon in the etch roughens the surface and there is evidence that plasmas produce substantial damage extending to thousands of angstroms below the surface (9). In identical samples, the reduced exposure time decreased the penetration depth but did not increase the peak concentration, indicating that diffusion played a larger role than damage in the interior hydrogen level (Fig. 7). No difference in zinc level was apparent.

The anneal temperature for which light output is increased within a few minutes fits well with OMVPE growth data (10). After growth of $p$-type InP/InGaAs/InGaAsP, the net acceptor concentrations are depressed. An anneal at $435{ }^{\circ} \mathrm{C}$ for $5 \mathrm{~min}$. in a non-hydrogen ambient reactivates the zinc. Fig. 6 shows the background level of this hydrogen after growth and anneal, before device processing.

QW structures were examined as well. They improved less than the bulk devices after annealing. This may be due to their reduced active region thickness ( $840 \AA$ vs. $1800 \AA$ for bulk), the fact they seemed to be less degraded in the first place and the high probability that the interfaces,

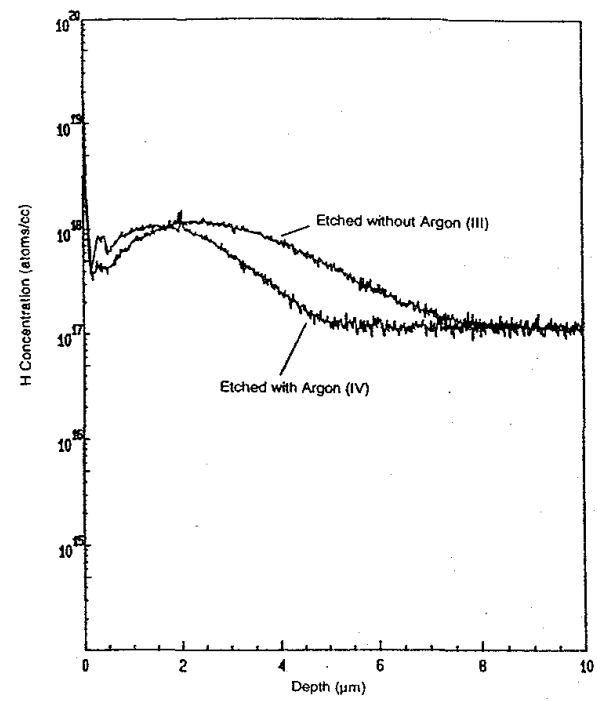

Fig. 7. Hydrogen SIMS data from samples etched without (III) and with Ar (IV) .

within the QW structure, are passivated. Hydrogen remaining at the interfaces after the anneal would be difficult to remove.

\section{Reliability Studies}

Reliability testing after the high temperature anneals was conducted on EELEDs and lasers at both constant power and constant current. Data from devices operated at $50{ }^{\circ} \mathrm{C}$ and $70{ }^{\circ} \mathrm{C}$ show that after a short "burn-in" interval of less than 100 hours, the current required to maintain a constant output power slowly decreased. At 7500 hours the required current had decreased by $2 \%$ of the initial value, leveled off and showed signs of increasing. Devices operating at $90^{\circ} \mathrm{C}$ exhibited a "burn in" followed by a short period of current decrease (to 1000 hours), followed by an increase in current.

Increasing power (decreasing current) is consistent with removal of residual hydrogen. Decreasing power (increasing current) is consistent with normal "wear-out" mechanisms. Although mechanisms compete and the length of each time period varies with temperature, all devices experience a "burn-in", hydrogen anneal and degradation (depicted schematically in Fig. 8). Results are similar for all samples whether capped with silicon dioxide or silicon nitride, annealed on a hot plate or in an RTA.

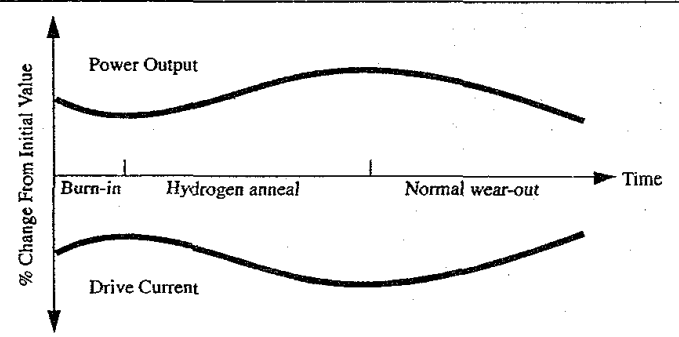

Fig. 8. General trend of reliability data (not to scale). 
These devices are normally operated at $20{ }^{\circ} \mathrm{C}$. A typical degradation will therefore not be observed until a time beyond the useful device life, generally $10^{4}$ hours.

\section{Discussion}

Hydrogen incorporation seems to become significant as wafers are cooled to $450^{\circ} \mathrm{C}$ after growth (10). This may explain why device output is not stable at elevated temperatures. A further anneal at $450{ }^{\circ} \mathrm{C}$ or above might rid the wafer of the last few percent, but could change the zinc placement. If zinc moves into the intrinsic active layer the device could be seriously degraded. The zinc profile following etching and after etching plus annealing is shown in Fig. 9. The zinc has moved toward the active region after only two minutes at $430{ }^{\circ} \mathrm{C}$.

We believe that free hydrogen electrically neutralizes the zinc doping $(11,12,13)$, degrading the contact and current confining properties of the ridge. Constricted current flow narrows the pumping region. Also, plasma damage can cause non-radiative recombination centers. These combined effects decrease device output power. Wider ridges have less overall change in output power because the hydrogen from the side walls and field area completely washes through a $4-\mu \mathrm{m}$ device while only a small percentage of a $100-\mu \mathrm{m}$ device is affected.

Photoluminescence measurements show a decreased peak intensity after the oxygen plasma clean-up which is necessary following methane-hydrogen etching. Polaron measurements of net active donor/acceptor concentration indicate only small changes in the minority carrier dopant in test structures and are inconclusive.

\section{Conclusions}

Hydrogen incorporation and an increase in output power after heat treatment has been observed in both bulk and quantum well active region devices emitting light at

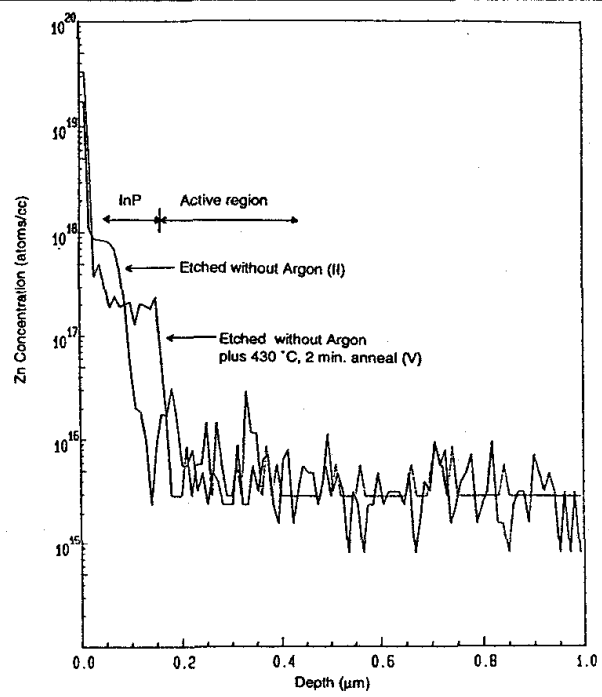

Fig. 9. Zinc SIMS data from samples II and V. various IR wavelengths. Anneals above $425{ }^{\circ} \mathrm{C}$ are effective in driving off hydrogen in a matter of minutes but they must be balanced with the movement of $p$ and $n$ dopants into the active region and degradation of other device layers, particularly metals. Anneals which balance time and temperature have been used to produce EELEDs with $1 \mathrm{~mW}$ output power and lasers with $20 \mathrm{~mW}$ of output power under normal operating conditions with minimal drift in reliability.

\section{Acknowledgments}

We thank Bill Perez for polaron and PL measurements, Dale Lefforge for SIMS and Karen Seaward, Mike Ludowise and N. M. Johnson for helpful discussions.

\section{References}

(1) K. Morit, et al., "Analysis of Damage During InP Hydrocarbon-RIE," Proc. of 4th Int. Conf. on InP and Related Materials, pp. 427-430, 1992.

(2) T. R. Hayes, et al., "Passivation of acceptors in $\mathrm{InP}$ resulting from $\mathrm{CH}_{4} / \mathrm{H}_{2}$ reactive ion etching," Appl. Phys. Lett., Vol. 55, No. 1, pp. 56-58, 1989.

(3) S. Cole, et al., "Effect of Cooling Ambient on Electrical Activation of Dopants in MOVPE of InP," Elect. Lett., Vol. 24, No. 15, pp. 929-930, 1988.

(4) S. Cole, et al., "Anomalous Behavior of Dopants in Atmospheric Pressure MOVPE of InP," J. Crystal Growth, Vol. 93, pp. 607-612, 1988.

(5) G. P. Agrawal and N. K. Dutta, Long Wavelength Semiconductor Lasers, Van Nostran Reinhold, N. Y., Sect. $5.4,1986$

(6) J. E. Schramm, "Reactive Ion Etching with $\mathrm{CH}_{4} / \mathrm{H}_{2} / \mathrm{Ar}$," Dept. of Elect. and Computer Eng. Rpt., U. C. S. B., 1993.

(7) J. Werking, et al., "Methane/hydrogen-based reactive ion etching of InAs, InP, GaAs and GaSb," Appl. Phys. Lett., Vol. 58. No. 18, pp. 2003-2005, May 1991.

(8) G. B. Stringfellow, Organometallic Vapor-Phase Epitaxy: Theory and Practice, Chapter 4.2, Academic Press, Boston, 1989.

(9) P. Viktorovitch, et al., "Passivation of InP for MISFET Applications," Proc. of 4th Int'l. Conf. on InP and Related Materials, pp. 51-55, 1992

(10) F. G. Kellert, et al., "Zn-doping in OMVPE Grown InP:Zn/InGaAs/InP $p-i-n$ Double Heterojunctions with InGaAs:Zn Contacting Layers," J. Elect. Mat., Vol. 21, No. 10, pp. 983-987, 1992.

(11) N. M. Johnson, et al., "Hydrogen passivation of shallow-acceptor impurities in p-type GaAs," Phys. Rev. B., Vol. 33, No. 2, pp. 1102-1105, Jan. 1986.

(12) W. C. Dautremont-Smith, et al., "Hydrogen Passivation of acceptors in $p$-InP." J. Appl. Phys. Vol. 66, No. 5, pp. 1993-1996, Sept. 1989.

(13) V. Swaminathan, "Hydrogen Passivation in III-V Compounds," Proceed. XII State of the Art Prgm. on Compound Semiconductors, Vol. 90-15, p. 20, Electrochem. Soc., 1990. 